\author{
Max Urchs
}

\title{
DEDUCTIVE MODELS AND PRACTICAL REASONING
}

\begin{abstract}
Cognitive psychology investigates the structural properties of human reasoning. We review some achievements of this kind of research and give a critical evaluation of its methods.
\end{abstract}

\section{CONTENTS}

1. Introduction, p. 150

2. Do we think deductively?, p. 151

3. Deductive abilities,p. 155

4. PSYCOP - an alternative system of deduction?, p. 159

5. An outlook, p. 163 
The study of deductive reasoning is the study of a psychological process. Although logical principles may be relevant to this process, they must be embodied as mental operations if they are to exert a direct causal influence on thinking.

L. J. Rips, The Psychology of Proof, p. 63

\section{Introduction}

The question whether or not deductive calculi are suitable models for human reasoning - or, more commonly: whether people (at least sometimes) reason deductively - is doubtlessly an interesting question per se. What is more, possible results of this kind of investigation contain the potential for far reaching applications. If there is a better understanding of the mechanisms guiding human thinking, then there are prospects for an improvement of these very processes. Understanding human reasoning makes it possible to teach people better thinking. Furthermore, for the time being human reasoning is the standard for the practically relevant drawing of inferences. This standard is not achieved by any non-human system. Even granted Deep Blue's spectacular success in playing chess, this is not what I mean by practically relevant inference making. From an anthropocentric perspective, the IBM-supercomputer appears to be much closer to an idiot savant than to a normally gifted individual. No construction from A.I. laboratories can compete with an average human with respect to universality and situation-dependant concentration on what is "really important". Human reasoning is still the only inference device what may be called "intelligent". Therefore investigations of this kind are of interest also from the point of view of Artificial Intelligence, which suffers from a chronic need of external inspiration. (That means, by the way, access to comfortable research programs.)

The question put forward at the beginning thus turns out to be scientifically interesting, ethically valuable, and commercially promising. It is obviously an invitation to interdisciplinary research. We try to approach problems like this in cognitive sciences. The logician looks over the fence of his own discipline into the neighbouring gardens, i.e. theoretical linguistics, cognitive psychology, A.I., psychological physics etc. 
Some of these interdisciplinary barriers are remarkably high, resembling walls rather than fences. The region between logic and psychology is an example. Because of historical prejudices, in this borderland neither logicians nor psychologists may be completely at ease. The rise of logicism under the influence of Frege and Husserl (see [7] and [8]) has meant the domination of anti-psychologism in logic ever since. This tendency is effectively complemented by analogous trends in modern psychology: in general, logic is perceived as no more than a methodological crutch useful for the design of experiments.

Without a doubt, this isolated development of both disciplines produced good results, in particular for the neat progress of logic. Yet currently open questions make any such dogmatic separatism increasingly conterproductive. That is perhaps why, among logicians as well as among some psychologists, there is a rethinking of possibly common fields of research. As far as this concerns logic, the reason might be the enormous difficulties with the development of inference mechanisms to be implemented in A.I. devices. It seems that the technical capacities of modern logic have come to an end here. That makes it natural to recall the unequaled efficiency of human reasoning in order to analyse that process and to receive inspiration.

Incidentally, that attitude leads one back to the roots of the WarsawLvov School. Some 100 years ago researchers there set out in harmonious balance between logical and psychological investigations. Later on, as is well known, the logical branch developed into the world-leading logical school, whereas the psychological one withered.

\section{Do we think deductively?}

Perhaps nobody expects me to give an explication of human thinking. This is certainly not the business of philosophy, not to mention logic. (Taking into account the versatile aspects and consequences of this question, there might well be no scientific answer at all, but theological, ethical or political answers instead.) The usual strategy in situations like this is to reduce the difficulties of the explication by simply assuming that people do in fact think from time to time. Then the question is: when may such an activity be called deductive?

Deduction is an ambious concept. First and foremost it means drawing inferences based on deductive rules, i.e. rules in which the consequences follow logically from the premises. In other words: they are correct with 
respect to some given logical calculus. A second meaning of the word denotes the deductive method, i.e. the method leading to the construction of deductive systems. In a third sense deduction means deductive thinking as opposed to reductive reasoning. In this last sense, deduction means to proceed from a reason to its consequence, along with the line of logical inference. Thus deductive reasoning divides (according to Jan Łukasiewicz) into deriving consequences (from reliable assumptions) on the one hand and testing hypotheses (searching for sure consequences of not perfectly certain assumptions) on the other hand.

For our purposes Łukasiewicz's conception of deduction splitting into derivations and testing hypotheses is not really interesting. Everybody is sometimes concerned with checking something or drawing inferences and thus with thinking deductively, according to the above definition. The question put foreward at the outset would thus gain a straightforward and quite irrelevant positive answer. The interesting problem is not to illustrate deductive reasoning by pointing to cases of deriving or testing something, but is found in a closer inspection of these very cases. How exactly does the underlying logical relation between assumptions and consequences look, what is the connection between the elements of the logical relation and the components of the mental operation, etc.?

For the time being, another classification of human mental operations seems to be more fruitful. Let us instead Ajdukiewicz's well-known multidimensional representation (see. [1]). Ajdukiewicz divides human reasoning a.o. into "deductive", "increasing probability", ..., "logically worthless". However the specific delimiting of these intervals may look, now the original question becomes interesting, since the first interval might turn out to be empty. Is that really the case? Certainly syllogistic reasoning belongs to deductive reasoning. So let us reformulate the question into a more specific one: Do we sometimes think in a syllogistic manner, according to the rules of syllogistic calculi? Maybe a practical example will be useful. We wish to transform the following syllogism into a first-order tautology:

No foxhound is a frog.

No cat is a foxhound.

No frog is a cat.

The problem, however, is this: the above syllogism is not a correct one. So there is no possibility to transform it into a tautology anyway. Following the line of the above reasoning, you may ask yourself whether you have had some uneasy feeling (aside from the usual stomachache of each humanist 
confronted with a syllogism.) Apparently we have a clear difference here to other cases of distorted harmony. Anybody will immediately notice the stain on a freshly ironed tablecloth, a piercing dissonance in a sonata, or a spoonful of Worcestershire sauce in your champagne. Our senses signal at once any of these deviations from the standard. As there is no analogue with logical disharmonies, it seems that our "logical senses" are not very well developed, or, to put it less speculatively: normally, people are not too good at reasoning. As experience shows, it is not hard at all to confuse your partner by stuffing your own argumentation with negations, temporal modalities or iterated conditional clauses. Taking into consideration probability claims makes the rate of possible errors (such as the gambler's fallacy, the principle of maximal meanness etc.) explode.

What is actually the reason for our assumption that people should be able to recognize a syllogism as correct or incorrect? Is it possible to acquire that skill the same way we learn a language? Or, is it true that when learning a language, we simultaneously learn the logic? How close is the analogy between natural language and formal logic? Does the logical competence of an individual depend on her experience, maybe even on anatomical peculiarities (similar to those which prevent the Chinese from speaking clear English)? Are there levels of logical competence within which unsound argumentation does not bother anybody nor even attract attention - again in analogy to natural language communication in groups using Saxonian or pidgin-English? Or do we "possess" our logic in a similar way as we own euclidean geometry? If so, why are there logical mistakes?

This gives rise to an important question: what actually is a logical mistake in concrete human reasoning? One can use this term either as a label for classifying specific results of empirical investigations on how human individuals draw inferences. Or one takes it as a valuation for judging and standardizing "wrong" answers during such tests. But what does it mean that a given answer is wrong? It means precisely that this answer does not correspond with what can be derived from the given premises according to the inference rules of some pre-established calculus. What logical calculus shall be the basis of such a classification? Usually the choice is classical propositional logic for propositional argumentation and traditional scholastic logic for syllogistic reasoning. That answer, however, seems far from being obvious: contemporary logic has a continuum of alternatives. Why should we choose exactly these two calculi? It is evident that the specific calculus, which is picked out to evaluate the answers, has a huge influence on the percentage of "true answers". Yet why should a person be counted as logically 
inferior only because she does not accept the S5-valid inference $\square \neg p$ on the basis of $\diamond \square \neg \diamond p$ or because she thinks she has good reason not to accept $H \vee G \vee G$ together with $H \vee G$ ? A possible compromise is offered by itself: by appropriate choice (or construction) of formal calculi one tries to model the results obtained during the empirical investigations. That would put us in a position to use logic as a meta-organon: the formal calculus would no longer be the standardizing ideal of real inference operations, but some tool that allowed us to classify the logical abilities of a person and thus to localize this person within a topography of agents performing inference operations. Some person would turn out to reason according to the modal calculus S4, while somebody else preferred, say, intuitionistic logic. We would thus arrive at some generalized version of Stelzner's hierarchy (cf. [18]).

There is a second problem, probably even deeper than the first. How can we give reasons for our silent assumption that formal systems might be authoritative for the outcome of human inference making? According to widespread opinion, specific logical calculi are to imitate idealizations of the autonomous processes of human reasoning. Keeping this in mind, logical mistakes turning up in empirical tests contradict rather the assumption of adequacy of the formal apparatus. In other words: can human reasoning be logically wrong at all? Is it not it simply the case that the logical systems, proposed as formalizations of human reasoning are not suitable and hence "defective"?

We have not yet mentioned what logic has contributed on its own. Evidently, the issue has obvious connections to the logical enterprise of natural deduction. Far from being unanimously defined, this concept denotes a large class of approaches aiming at rule-based descriptions of logical calculi. It is not an invention of our days. Historians of logic suspect the genesis of natural deduction already in Aristotelian syllogistics and in Stoic logic where the deduction theorem was used in praxis. Anyway, what we do have here are examples of using that kind of technique, without even seeing the need of giving reasons for them. This work began only in 1926, when Łukasiewicz stated the problem of a formal reconstruction of theorem proving in mathematical praxis. These practical demonstrations of mathematical truths were far away from what counted as a proof in logic. Proofs by assumptions, indirect proofs or alternative cases in the course of a proof all need a theoretical elucidation. As a side effect of these investigations one might hope for a formally correct and more feasible method of proof in logic, since proving theorems within axiomatic systems is quite a hard job. There is a common origin of almost all constructions within this realm which are directed to- 
wards possible practical investigations, namely Jaśkowski's work on natural deduction. (Gentzen's alternative approach aims rather at proof-theoretic aspects.) Helpful as the technical work carried out by logic turns out to be - as we will see in due time - it will definitely not provide us with straightforward answers to all the questions mentioned above. Logic has nothing essential to say about its relation to non-logical fields.

This seems to be the right moment to take a look over the fence. How does a cognitive psychologist, sympathetic to logical investigations, approach these problems?

\section{Deductive abilities}

It is an interesting task to investigate specific cases in which people reason logically. This problem has many aspects. One of them is the question whether individual human beings reason in the same way as groups of people do. What about e.g. large groups of people acting collectively? Is there any chance to analyse their behavior alongside the analysis of individual human's behavior? It is easy to find extremely sceptical views on the matter. Let us quote from Schopenhauer's "Erystic" (about 1830):

Indeed, there is no opinion so absurd that people would not be ready to take it as their own, if only one manages to persuade them that this very opinion is held by the general public. An example influences their thought, as well as their action. They are like sheep who follow their bell-wether, wherever he leads them; they would rather die than think.

If Schopenhauer (not to mention many others) is right, then there is not much to analyse here: they just do not think autonomously and hence they do not think deductively. But, that might still leave a loop-hole for logical analysis. What a large group of agents is going to do seems to be significantly influenced or even preestablished by the by the behavior of its leader. The idea could be then to take the leader as the designated element which represents the behavior of the whole group. Yet a second thought renders this way out rather impossible as well: people are the more willing to follow some leader the more irrationally he behaves. ${ }^{1}$

So the logic of human communities seems to be a fuzzy task for, perhaps, sociology to cope with. In experimental psychology, researchers are instead

\footnotetext{
${ }^{1}$ Schopenhauer's picture turns out to be adequate also in this respect: in general people choose a bell-wether to follow rather than a shepherd.
} 
interested in measuring the logical abilities of concrete human individuals. For that purpose, one usually asks a testgroup of people to decide whether a couple of given syllogisms or propositional rules are logically correct. Subsequently one determines the rate of true answers per group. Thereby for every logical figure is established its rate of acceptance. Answers deviating from the standard may have various explanations. Given a rule $P / G$ which is correct for a calculus $T$, let us suppose that a test person $x$ does not infer a conclusion of the form $Q$ from premises of the form $P$. The reason for that may be one of the following:

- $x$ does not accept the calculus $T$

(only reasons "internally" according to some $T^{\prime}$ different from $T$ );

- $x$ understands the schema in question erroneously as the formalisation of a plausible inference

( $H \rightarrow F, \neg H / \neg F$ is associated with "Whoever has the right kind of ticket may use the Intercity. Mr. X again has no excess fare, hence he is not allowed to board the train"; "If somebody spends more than $\$ 50$, she gets one T-shirt with the shop's logo free. This lady only tried on several things, therefore she won't get one of our T-shirts.");

- $x$ mistakes $P / Q$ for a structurally similar but correct schema (e.g. $P \rightarrow Q \vee R$ for $P \rightarrow Q \wedge R$ with respect to the consequence $P \rightarrow Q$ );

- very practical obstacles are in the way of the correct answer (shortage of time, problems with concentration);

- $Q$ is refused by $x$ for ethical reasons (e.g. because it contains obscene words, or because the utilization of formal means is generally condemned in the given context);

- $x$ does not understand that she is supposed to judge the derivability of $Q$ (but considers $Q$ 's plausibility instead, or its probability);

- $x$ is subject to misleading suggestions during the test

(An "unfair" experimenter assigns the task: "Show that ...", where there is actually nothing to show, since the schema is incorrect, or just the other way round: "Disprove that ...", where the schema is correct.)

- $x$ does not want to admit that the task exceeds his skills and therefore tries to guess, unfortunately without good luck ...

This list may be extended. After analyzing the given answers as carefully as possible (which should lead to the exclusion of non-logical disturbances 
among the mistaken answers) schemata with very low rates of acceptance during the tests - although being logically correct in the formal calculus should perhaps be excluded from the "natural logic". As a typical result, one finds that the syllogism

$$
\begin{aligned}
& \text { All apes like sugar. } \\
& \text { Some animals are apes. } \\
& \text { Some animals like sugar. }
\end{aligned}
$$

is recognised as true by $100 \%$ of the test persons, whereas negation provides the usual difficulties:

$$
\begin{aligned}
& \text { All apes like sugar. } \\
& \text { Some intelligent beings are not apes. } \\
& \text { Some intelligent beings don't like sugar. }
\end{aligned}
$$

On the basis of such investigations one later obtains interesting observations and generalizations, e.g. the atmosphere hypotheses of Woodworth and Sells from 1935: existential premises have a tendency to cause strong preferences for existential consequences (and quite similar for negated premises). Furthermore it shows - and is widely used in propaganda - that a person is less critical towards arguments whose consequences are sympathetic for some reason or other. This way one obtains a couple of interesting hypotheses, but hardly a complete theory of human deductive behavior. Some of the summarizing thoughts of such experiences are so deeply sad that they are quite amusing:

Our evidence will indicate that the only circumstances under which we can be relatively sure that the inferences of a person will be logical is when they lead to a conclusion which she has already accepted. [12, p. 39]

Empirical investigations of inference behavior sometimes seem to be almost chaotic. The following findings are quite representative: Neither a one-term class in formal logic nor detailed instructions on how to transform negated quantified sentences improves the results of a given testgroup, whereas intensive training raises the percentage of correct answers about equivalent conditionals from $27 \%$ to $81 \%$. Logic courses improve the logical abilities of law and psychology students, but do not do any good for students of philosophy and chemistry ([11], [13]). Sometimes one has the impression that it is not at all clear what properties are measured by these tests. The more so since during some of the experiments the slightest changes in the setting lead to dramatically modified outcomes ([9]). 
As the saying goes, there are no limits to logical incompetence. But even then it could make sense to count the acceptance rates for logical schemata and subsequently to distinguish the figures with high scores as the ones which form the natural logic. But doesn't such a procedure mean sacrificing the standard of universal validity of logical rules. Doesn't it lead to a conception of truth (of logical objects!) according to majority resolutions, to some postmodern "truth by handwaving"? And what, precisely, one is supposed to measure by such experiments?

It does not seem at all obvious whether by these investigations such a thing as "natural logic" is uncovered. I am rather inclined to think that the afore-mentioned means of research are entirely inappropriate. To my mind, it resembles an expedition of lion hunters who try to catch the king of the desert by systematically sifting tons and tons of sand. The logic hunters are in a relatively worse position insofar as they do not even know whether the object of their search exists. Maybe it was the dread of such an evolutionary logic what made Frege and Husserl, in their day, burn down the bridges to psychological aspects of logic altogether.

Let us come back to the idea of treating the manifold of logical calculi as a tool for exterior localization of the deductive abilities of human individuals. Admittedly one has to formulate very carefully here. Imagine that a person has perfect command of syllogistic logic. He solves all tasks from that problem area without any mistakes and does so "purely mentally", i.e. without paper and pencil, without gesticulation or murmuring during his work. In that situation one has to acknowledge that this person possesses an internal algorithm to solve this kind of problem. This algorithm, however, can hardly be seen as a "tool", as part of the logical organon. The algorithm should thus be accepted as being part of the person's style of thought (in Łukasiewicz's sense: logic as the aesthetics of speech and thought).

Theoretically this leaves us with the possibility of a conspiracy theory in the foundations of logic: mean teachers consciously instruct their students with inappropriate rules. As a matter of fact, some blame towards the traditional Collegium Logicum come from that direction: acquired faulty routines of theorem proving leads to wrong strategies in problem solving. This seems to be slightly exaggerated - intentional brain-washing is surely not the main source of ubiquitous failures in practical reasoning. 


\section{PsyCOP - an alternative system of deduction?}

Deductive reasoning in normal-life situations demands huge resources in a system's memory and calculation speed. According to widespread opinion in robotics, in the near future no can? on wheels, reasoning purely deductively, will be able to safely cross a busy street. On the other hand, there is nothing better for algorithmic, i.e. rule-based problem solving than the deductive method. So investigations of this issue have been conducted for many years.

Early approaches began at the beginning of the century. This period of investigations of easy syllogisms ended with results like the atmosphere hypotheses, mentioned above. A second stage began in the 1960s together with the evolution of artificial intelligence. It is characterized by more sophisticated formal methods which allow us to consider forms of reasoning other than syllogisms. There were plenty of new results, but no overlapping theory.

Theorists seem simply to write a different theory for each task. [...] There is no way to relate them and thus they help to ensure the division of the study of human cognition into qualitatively isolated areas. [14, p. 39]

In a third phase of psychological investigation on human reasoning all of the relevant activities were treated as special forms of one specific human ability: problem solving. Unfortunately, from this perspective a deductive inference is as good an object for investigation as are chess riddles or crypto-arithmetic puzzles. The leading conception is best represented by Allen Newell's and Herbert Simon's classical General Problem Solver. This formal conception came together with a philosophical attitude. As it is well-known, the Church-Hypotheses proposes to identify human calculating abilities with the respective abilities of a Turing machine. Newell and Simon took a large step forward: They had a new answer to the old question "What is a man?" The characteristic property is the ability of manipulating abstract symbols. Consequently, the essence of man is nothing else but a Turing machine. ${ }^{2}$

D. Osherson's approach (cf. [16]) leads to the development of so-called "natural logic" by Braine and others (see [2], [3]). This conception is characterized by additional rules to handle more than one argument, to introduce

\footnotetext{
2 Then, however, recursion theory becomes a kind of protopsychology. Moreover, Turing machines with oracle turn out to be interesting character studies of badly organized contemporaries and to be completely drunken means nothing but the failure of the reading and printing device together with partial loss of storage tape ....
} 
suppositions and react to encountered inconsistencies. In the first phase, the strategy follows the usual British Museum algorithm. ${ }^{3}$ If this does not lead to the conclusion or to a contradiction, one tries in a second phase to close the proof by moving backwards. Unfortunately, the technical performance is not perfect; sometimes it falls into loops. Also other constructions are somewhat defective according to logical standards. So-called selection tasks are treated by "pragmatic schemata", i.e. by "a set of generalized, context-sensitive rules which, unlike purely syntactic rules, are defined in terms of classes of goals (such as taking desirable actions or making predictions about possible future events) and relationsships to these goals (such as cause and effect or precondition and allowable action)" [4, p. 395]. However, the resulting deontic calculus has a peculiar property: "the rules attached to reasoning schemas are often useful heuristics rather than strictly valid inferences" [4, p. 397].

Among other propositions one finds Cosmides' conception of social contracts (see e.g. [5]), or non-deductive mental models like those of JohnsonLaird and Byrne (cf. [10]).

In the eighties, L. J. Rips developed through several stages the system PSYCOP (for: Psychology of Proof), which exists as a PROLOG-programm as well. The author puts the main idea of the construction this way: "we get a satisfactory account of human deduction by marrying the notion of a supposition from formal natural deduction systems in logic with the notion of a subgoal from models of problem solving in artificial intelligence." [17, p. 104] The system consists of two large classes of rules. The first one contains 10 inference rules (with sometimes multiple variants) working from premises towards conclusions. The second class has 14 members, which are called strategy rules. These rules work backwards in a sense. Setting out at the conclusion, they establish subgoals, which are to be achieved subsequently using rules from the first group. There exists a first-order variant of PSYCOP, too. For that purpose both groups of rules are enlarged by three further inference rules and 14 new strategy rules, in addition to four rules regulating the substitution of free variables. Here is an example of an inference rule, the so-called rule of Forward Conjunctive Modus Ponens:

\footnotetext{
3 "To prove that a given conclusion follows from a set of premises, start by writing down a list of the premises. Apply the rules to the premises, and add any new sentences they produce to the premise list. If the conclusion is on this list, then the algorithm halts and the conclusion has been proved. Otherwise, apply the rules once again to the enlarged list of sentences, adding any new items to the premise-and-derived-sentence list. Continue in this way until the conclusion is proved." [15]
} 


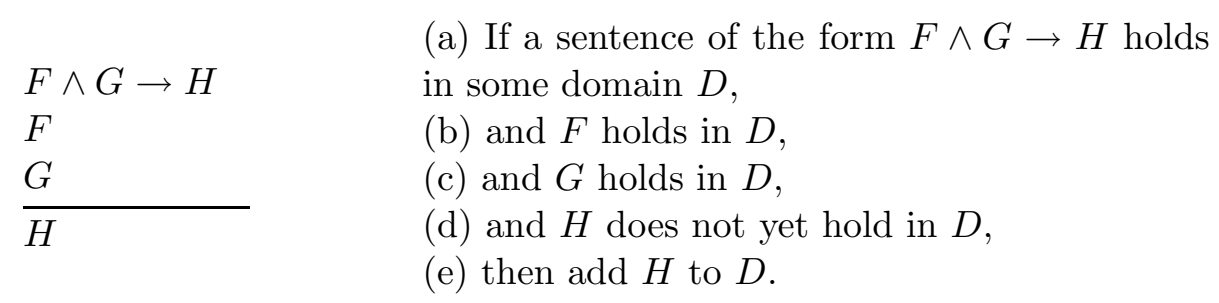

A typical strategy rule is the following Backward Double Negation Elimination:

(a) Set $D$ to domain of current goal,

(b) Set $H$ to the current goal,

$\frac{\neg \neg H}{H}$

(c) If the sentence $\neg \neg H$ is a subformula of a sentence that holds in $D$,

(d) and $D$ does not yet contain a subgoal $\neg \neg H$,

(e) then add $\neg \neg H$ to the list of subgoals.

All inference as well as all strategy rules constituting the calculus came out during appropriate experiments as deductive figures which are used in practical reasoning. That means that all these rules were recognised as correct and were used by subjects in further deduction with some reasonably high certainty. Remarkably, all the rules in PSYCOP are correct in classical logic. Keeping in mind the popularity of certain incorrect rules in practical reasoning (e.g. from $F \rightarrow H$ and $\neg F$ to $\neg H$ ) this is by no means obvious and even somewhat inexplicable. Did the authors tacitly use a second filter in the construction of PSYCOP, namely the classical correctness of schemata?

By the "specific probability" of an inference schema we understand the percentage of true answers classifying it as a correct rule during the tests. According to the specific purpose of the calculus it is no fault that the rules are not independent. Interestingly enough, they are not complete, either. In order to achieve classical completeness, one has to add a conditional transformation

$$
\frac{F \rightarrow H}{\neg F \vee H}
$$

or else a negated conditional transformation

$$
\frac{\neg(F \rightarrow H)}{F, \neg H}
$$

(which do not belong to PSYCOP). Now we are in a position to ask several questions about this logical system, e.g. whether it is decidable etc. 
The really interesting observations concern the problem of how proof routines carried out in PSYCOP correspond with the behavior of specific individuals solving the given tasks. That, after all, was why PSYCOP was constructed. This is done as follows: The test persons get an inference figure $R$ and one counts the rate of answers classifying it as "correct" or as "not correct". If a test person is unable to give an argument in favor of one answer or the other, she should guess. Subsequently one determines the rate of true answers per test group with respect to this figure. The next step consists of an analysis of the proof-tree for $R$ in PSYCOP.

Just to give an example, let us suppose that the rules $r_{1}, r_{2}$ and $r_{3}$ are used in order to perform some proof. All rules in PSYCOP are distinguished by psychological adequacy, which is expressed by some high rate of their acceptance, i.e. by high specific probability. In particular, for each of these rules, its specific probability is known. The probability $P(R)$ of $R$ is established by the formula:

$$
P(R)=p_{1} \cdot p_{2} \cdot p_{3}+0,5 p_{g}\left(1-p_{1} \cdot p_{2} \cdot p_{3}\right)
$$

where $p_{g}$ denotes the probability according to which a test person tries a guess. In some special cases there might exist an alternative proof tree for $R$, using the rules $r_{1}, r_{2}, r_{4}$ and $r_{5}$. In that situation, $R$ 's probability equals

$$
\begin{aligned}
P(R)= & p_{1} \cdot p_{2} \cdot p_{3}+\left(1-p_{3}\right) p_{1} \cdots p_{2} \cdots p_{4} \cdots p_{5}+ \\
& \left.+0,5 p_{g}\left(1-p_{1} \cdot p_{2} \cdot p_{3}\right)-\left(1-p_{3}\right) p_{1} \cdots p_{2} \cdots p_{4} \cdots p_{5}\right)
\end{aligned}
$$

The subjects got 32 correct inference schemata, 32 incorrect ones, and "for relaxation" 40 filler arguments (not included in the rating). The correspondence of values for answers predicted this way and experimentally observed answers is sometimes impressive, indeed. In the case of the 32 correct schemata, one found for instance the following (typical!) relations between calculated and measured values:

$\begin{array}{lcc}\text { Argument } & \text { Observed } & \text { Predicted } \\ F & 66.7 & 70.2 \\ \frac{H \vee G}{\neg H \rightarrow G \vee F} & & \\ \frac{(F \rightarrow H) \wedge F \wedge G}{H \wedge G} & 80.6 & 76.6 \\ \frac{F \vee H \rightarrow \neg G}{F \rightarrow \neg(G \wedge K)} & 50.0 & 38.1\end{array}$




$$
\begin{aligned}
& F \\
& F \vee H \rightarrow G \\
& G \rightarrow K \\
& \hline K \vee L
\end{aligned}
$$

Rips thinks about the development of PSYCOP as a universal deductive theory, containing e.g. rules for modalities (the ghost of mathesis universalis seems to be invincible.)

\section{An outlook}

What are the results of our "stocktaking" of logical investigations concerning human reasoning within psychology? The situation we have met resembles the one of an earlier excursion to artificial intelligence (cf. [19]). The specialists investigate classes of systems which are well known in logical literature for a couple of years. The logician finds with a blend of amusement and respect that parts of the logical apparatus, relevant to the problems under investigation, are, so to say, reinvented on overdrive. This way highly interesting examples, metaphors and formulations appear. ${ }^{4}$ Rips' above mentioned monograph ([17]) ends in 1994 somewhere between non-monotonic reasoning and knowledge revision. As a hole, one is convinced about ones own approaches; critical remarks are hardly ever noticed. In Rips, for instance, there is no discussion at all of the fundamental critique of the possibility of a description of human thinking by means of abstract symbol manipulation. ${ }^{5}$ In logical psychology, one prefers to stay in the realm of what John Haugeland called Good Old-Fashioned AI.

All this leads to the conviction that these investigations of psychologists are - from our point of view - the far less interesting part of the contribution of psychology. Even if it were possible to develop a deductive calculus widely applicable to human reasoning (and, PSYCOP is a fairly promising candidate for that aim), this would not yield any of the expected essentially new stimulation for logic and artificial intelligence. What really is of interest is not the work of the logically inclined psychologist qua logician, but

\footnotetext{
${ }^{4}$ There are curious examples revealing the different standards in both psychology and in logic. For instance, when Rips gives no less than four references to prove the elimination of double negation in classical, while being incorrect in intuitionistic logic. [17, p. 378]

5 "After fifty years of effort, however, it is now clear to all but a few diehards that [...] the research program based on the assumption that human beings produce intelligence using facts and rules has reached a dead end, and there is no reason to think it could ever succeed." [6, p. ix]
} 
qua psychologist. It is far from obvious whether - in order to get findings about hidden thought processes - one should directly model empirical data by logical means, or to try to better understand these processes through further psychological analysis and neurological investigations first. (It might be very enlightening, e.g. to understand the transition from deduction to reasoning by means of mental "pictures", which in some situations seems to be much a more effective method of problem solving.)

One has to confess, of course, that similar questions are far away from getting precise and useful answers. Contemporary investigations concern problems of how the most primitive organisms realize very simple memory processes. The favorite object of investigations is Aplysia Californica, a naked snail with altogether 20.000 neurons. That is the amount contained in one thousandth of a gram of human brain substance.

The methodology of experimentation on the open human brain seems to be not yet fully developed. The extreme complexity of the matter can hardly be overestimated. For example, in order to find out the relevant areas in brain surgery one encircles them by touching some place on the cortex and asking the patient what effect this has. Some neurosurgeons are astonished by the observation that touching "the same point" of the cortex twice brings about quite different reminiscences from e.g. the patient's childhood. This recalls the traveller who wonders why during each visit to N.Y.C. he meets different people on Times Square. The only encouraging fact I can see so far follows from Rips' investigations on the PSYCOP system. One has the clear impression that here one found some regularity. In other words, one must probably accept a hidden connection between the psychological process of deductive reasoning and a formal calculus which by far exceeds casual correlation.

One conclusion is certainly reasonable: the investigation of the mechanism of human thinking is par excellence an interdisciplinary problem. As such, it should be best advanced within cognitive science.

\section{References}

[1] Ajdukiewicz, K., "Klasyfikacja rozumowań", Studia Logica 2 (1955), 278-299.

[2] Braine, M. D. S., "The 'natural logic' approach to reasoning", in: Overton (ed.), Reasoning, Necessity, and Logic: Developmental Perspectives, Erlbaum 1990.

[3] Braine, M. D. S., B. J. Reiser, and B. Rumain, "Some empirical justification for a theory of natural propositional reasoning", in: Bower (ed.), Psychology of Learning and Motivation, vol. 18, Academic Press 1984. 
[4] Cheng, P. W., and K. J. Holyoak, "Pragmatic reasoning schemas", Cognitive Psychology 17 (1985), 391-416.

[5] Cosmides, L., "The logic of social exchange: Has natural selection shaped how humans reason?", Cognition 31 (1989), 187-276.

[6] Dreyfus, H. L., What Computers Still Cant't Do. A critique of artificial reason, The MIT Press Cambridge, Mass., London 1992.

[7] Frege, G., Grundgesetze der Arithmetik, Jena 1893-1903.

[8] Husserl, E., Logische Untersuchungen, Halle 1900-1901.

[9] Jackson, S. L., and R. A. Griggs, "The elusive pragmatic reasoning schemas effect" ,Quarterly Journal of Experimental Psychology 42A (1990), 353-373.

[10] Johnson-Laird, P. N., and R. M. J. Byrne, Deduction, Erlbaum 1991.

[11] Lehman, D. R., R. O. Lampert, and R. E. Nisbett, "The effects of graduate training on reasoning", American Psychologist 43 (1988), 431-442.

[12] Morgan, J. J. B., and J. T. Morton, "The distortion of syllogistic reasoning produced by personal convictions", Journal of Social Psychology 20 (1944), $39-59$.

[13] Morris, M. W., and R. E. Nisbett, "Tools of the trade: Deductive schemas taught in psychology and philosophy", in: Nisbett (ed.), Rules for Reasoning, Erlbaum 1993.

[14] Newell, A., "Reasoning, problem solving, and decision process. The problem space as a fundamental category", in: R. S. Nickerson (ed.), Attention and Performance VIII, Erlbaum 1980.

[15] Newell, A., J. C. Shaw, and H. A. Simon, "Empirical Explorations with the Logic Theory Machine: A case study in heuristics", in: Proceedings of the Western Joint Computer Conference 1957.

[16] Osherson, D. N., Logical Abilities in Children, vol. 1-4, Erlbaum 1974-1976.

[17] Rips, L. J., The Psychology of Proof. Deductive Reasoning in Human Thinking, MIT Press 1994.

[18] Stelzner, W., Epistemische Logik, Akademie Verlag, Berlin 1984.

[19] Urchs, M. P., "Artificial causality", in: Faye, Scheffler, Urchs (eds.), Logic and Causal Reasoning, Akademie Verlag, Berlin 1994.

MAX URChS

Department of Logic

N. Copernicus University

ul. Asnyka 2

31-044 Torun, Poland

e-mail: max@mat .uni.torun.pl 